130.6

\title{
ON THE EVALUATION OF SPATIAL DOMAIN MOM MATRIX ENTRIES CONTAINING CLOSED FORM GREEN'S FUNCTIONS
}

\author{
Noyan Kınayman ${ }^{1}$, M. I. Aksun ${ }^{2}$, and R. Mittra ${ }^{3}$ \\ ${ }^{1,2}$ Bilkent University, EE Engineering Department, Ankara 06533, TURKEY \\ ${ }^{3}$ Pennsylvania State University, EE Engineering Department, PA 16802-2705, USA
}

\section{Introduction}

The Method of Moments (MoM) is one of the widely-used numerical techniques employed for the solution of Mixed Potential Integral Equations (MPIE) arising in the analysis of planar stratified geometries, e.g., MMICs. However, the application of this technique in the spatial domain poses some difficulties since the associated spatial-domain Green's functions for these geometries are improper oscillatory integrals, known as Sommerfeld integrals [1,2], that are very computationally-intensive to evaluate. It is possible to eliminate the time-consuming task of computing these integrals by using closed form versions of the spatial domain Green's functions [3, 4], and the time required to evaluate the reaction integrals in the MoM matrix can be reduced considerably. Furthermore, it has been shown recently that the reaction integrals resulting from the application of the MoM can also be evaluated analytically by using piecewise linear basis and testing functions [5]. Hence, an efficient EM simulation algorithm can be developed by using the closed form Green's functions in the MoM formulation that involves no numerical integration.

However, despite the time-saving realized from the analytical evaluation of the reaction integrals with the closed-form Green's functions, the need for further reducing the matrix fill-time is not obviated for many problems. With this background in mind, the objective of this paper is to present a hybrid technique for the evaluation of the MoM reaction integrals in a numerically-efficient manner that further reduces the time need for their computation.

\section{MPIE Formulation and MoM Matrix Entries}

To generate the MoM matrix we follow the MPIE formulation, and express the tangential components of the electric field in a planar geometry in terms of the surface current density $\mathbf{J}$, and the associated Green's functions of vector and scalar potentials, as follows [6]:

$$
\mathbf{E}=-j w\left(G_{x x}^{A} * J_{x}\right) \hat{\mathbf{x}}-j w\left(G_{y y}^{A} * J_{y}\right) \hat{\mathbf{y}}+\frac{1}{j w} \nabla\left(G^{q} * \nabla \cdot \mathbf{J}\right)
$$

where * denotes convolution. The term $G_{i j}^{A}$ represents the $i$-directed vector potential at $\mathbf{r}$ due to an $j$-directed electric dipole of unit strength located at $\mathbf{r}^{\prime}$, while $G^{q}$ is the scalar potential due to a unit point charge associated with an electric dipole. Next, we follow the usual procedure to generate the MoM matrix whose typical entry is given by:

$$
\left\langle T_{x m}, G_{x x}^{A} * J_{x n}\right\rangle+\frac{1}{w^{2}}\left\langle T_{x m}, \frac{\partial}{\partial x}\left[G_{x}^{q} * \frac{\partial J_{x n}}{\partial x}\right]\right\rangle
$$

0-7803-4178-3/97/\$10.00 C 1997 IEEE 
where $T_{x m}$, and $J_{x n}$ denote the testing and basis functions, respectively, and $\langle$, designates the inner product. The spatial domain Green's functions in (2) are the closed form types, that have the generic form:

$$
G^{A, q} \cong \sum_{n=1}^{N} a_{n} \frac{e^{-j k_{i} r_{n}}}{r_{n}}
$$

where $r_{n}=\sqrt{\rho^{2}-b_{n}^{2}}$, and $\rho=\sqrt{x^{2}+y^{2}}$. The methods for deriving the closed-form spatial-domain Green's functions have been sufficiently detailed in the literature $[3,4,7]$ and, consequently, are omitted here.

\section{Evaluation of the MoM Matrix Entries}

Efficient evaluation of the MoM matrix entries given in (2) is an important issue that deserves our attention, because it is the principal contributor to the total CPU time for small and moderate-size geometries. The MoM matrix entries can be calculated analytically without any numerical integration for piecewise basis and testing functions via the rigorous approach described in [5], provided the closed form Green's functions are used for the formulation. In the above method, each of the exponentials in (3) is replaced by its Taylor series approximation as follows:

$$
G^{A, q} \cong \sum_{n=1}^{N} a_{n} \sum_{m=0}^{M} c_{m} \frac{\left(r_{n}-r_{c}\right)^{m}}{r_{n}}
$$

where $c_{m}$ 's are the Taylor series coefficients and $r_{c}$ is the center of expansion for the exponential term $e^{-j k_{i} r_{n}}$. Alternatively, one could replace the entire Green's function in (3) with a suitable approximation that enables him to compute the reaction integrals analytically. For instance, one may use the polynomial approximation for the Green's function given by:

$$
G^{A, q} \cong \sum_{l=-1}^{L} b_{l} \cdot \rho^{l}
$$

where $b_{l}$ 's are the complex coefficients obtained through a least-square fitting scheme. It is evident that the analytical integration of the reaction integrals is considerably simpler when the Green's functions is expressed as in (5), rather than when it is expanded as in (4). This is because the analytical evaluation of the inner-product integrals using the former representation requires extensive complex arithmetic operations, as well as multiple evaluations of complex logarithms and trigonometric functions [5]. However, the caveat in the polynomial-fitting approach is that approximating the entire Green's function is very difficult, if not impossible, with a relatively small $L$, because of the singular behavior of the Green's functions as $\rho \rightarrow 0$. One approach to resolving this dilemma is to utilize both of the above representations, but in complementary regions, thereby taking advantage of the salutary features of both. This can be done by using the (4) to represent the Green's function for small $\rho$, where it exhibits a singular behavior, and then switch over to the (5) as $\rho$ becomes larger.

To summarize, a direct application of the rigorous method places an unnecessary computational burden when $\rho$, the distance between the source and observation 
points, is greater than a predetermined value $\rho_{l s}$. To circumvent this problem, one can use a hybrid approach, whose flow chart is shown in Fig. 1, that uses a judicious combination of the two methods to increase the computational speed with which the MoM matrix entries are generated. At this point it is worthwhile to describe the strategy for employing the hybrid technique. To be able to use a smaller $L$ in (5), the polynomial-fitting scheme should be carried out over a small range of $\rho$, and this requires that the least-squares fitting be repeated for each of the inner-product operation. Consequently, to accelerate the fitting process, the Green's function can be sampled between $\rho_{l s}$ and $\rho_{\text {max }}$ and stored in a look-up table before starting to fill the MoM matrix. These tabulated values can be subsequently interpolated to perform the least-squares fitting relatively quickly for each inner-product operation.

\section{Results and Conclusions}

A microstrip patch antenna, shown in Fig. 2, has been analyzed to demonstrate the efficiency and accuracy of the hybrid method. As a first step, we investigate the effect of the choice of $\rho_{l s}$ on the matrix fill-time. The number of basis functions for the patch antenna is chosen to be 537, and the CPU times, shown in Fig. 3, are obtained as the auxiliary parameter $s$ is varied. Note that the rigorous method, which is used here as the reference, corresponds to $s=\infty$. Next, we investigate how the choice of $\rho_{l s}$ affects the accuracy of the results. The plots shown in Figs. 4 and 5 show the impedance of the patch antenna derived by using different values of $s$. We observe that, for this geometry, reducing $\rho_{l s}$ below a certain value introduces unacceptable errors in the results.

We conclude with the observation that the proposed hybrid method can reduce the matrix fill-time significantly, without sacrificing the accuracy, with an appropriate choice for $\rho_{l s}$. In addition, we find that the proper choice of $\rho_{l s}$ depends upon the Green's function, as well as on the cell size used for discretization, and that the choice of $\rho_{\max }$ should be carried out in accordance with the magnitude of the Green's function at $\rho_{\max }$.

\section{References}

[1] A. Sommerfeld. Partial Differential Equations in Physics. Academic Press Inc., 1949.

[2] W. C. Chew. Waves and Fields in Inhomogeneous Media. Van Nostrad Reinhold, 1990.

[3] Y. L. Chow, J. I. Yang, D. G. Fang and G. E. Howard "A Closed-Form Spatial Green's Function for the Thick Microstrip Substrate," IEEE Trans. on Microwave Theory Tech., vol. 39, pp. 588-592, March 1991

[4] M. I. Aksun and Raj Mittra "Derivation of Closed-Form Green's Functions for a General Microstrip Geometry," IEEE Trans. on Microwave Theory Tech., vol. 40, pp. 2055-2062, Nov. 1992.

[5] Lale Alatan, M. I. Aksun, Karthikeyan Mahadevan, and Tuncay Birand "Analytical Evaluation of the MoM Matrix Elements," IEEE Trans. on Microwave Theory Tech., vol. 44, pp. 519-525, April 1996.

[6] Juan R. Mosig "Arbitrarily Shaped Microstrip Structures and Their Analysis with a Mixed Potential Integral Equation," IEEE Trans. on Microwave Theory Tech., vol. MTT-36, pp. 314-323, February 1988.

[7] M. I. Aksun "A Robust Approach for The Derivation of Closed-Form Green's Functions," IEEE Trans. on Microwave Theory Tech., vol. 44, pp. 651-658, May 1996. 


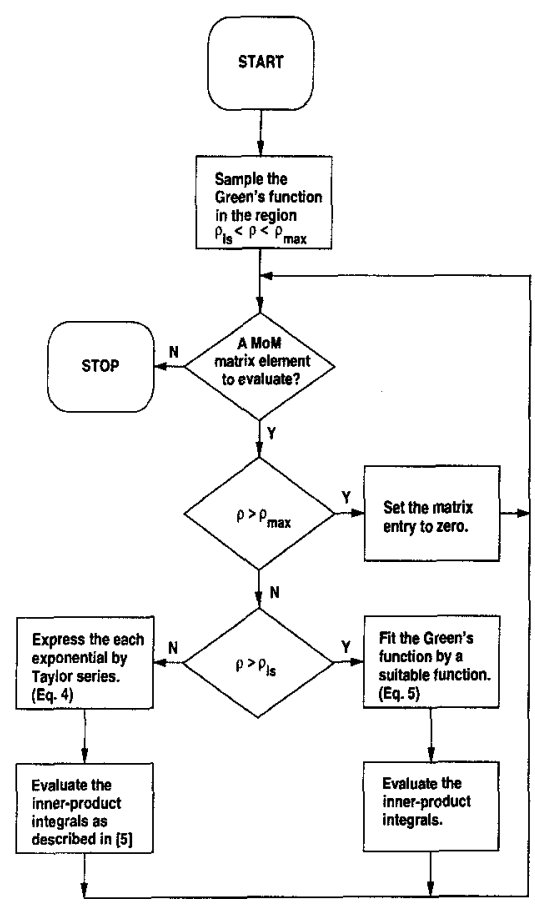

Figure 1: Flow chart of the proposed hybrid method for evaluating the MoM matrix entries.

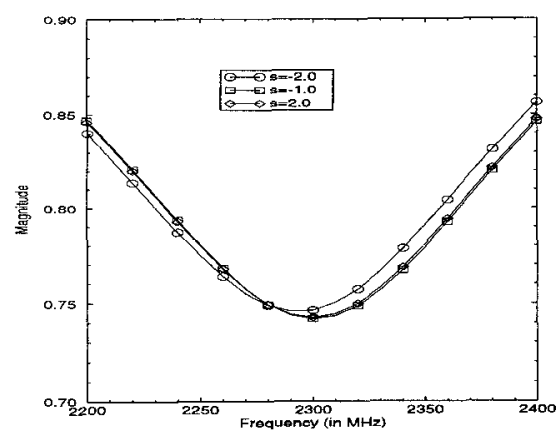

Figure 4: Magnitude of the input impedance of the patch antenna.

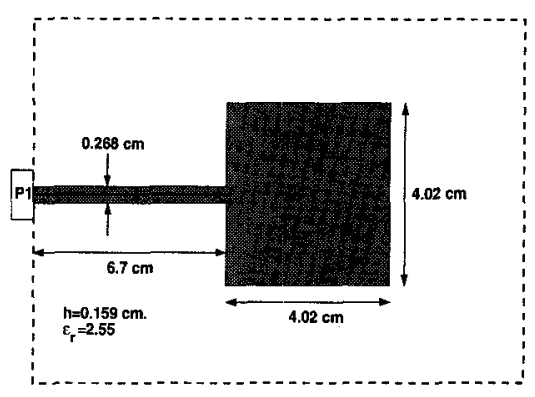

Figure 2: Geometry of the microstrip patch antenna (not to scale).

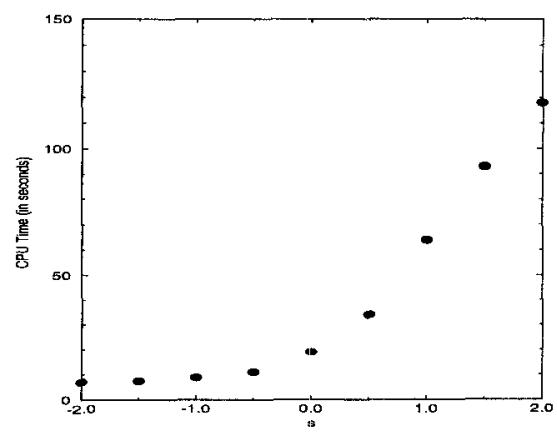

Figure 3: CPU times vs, auxiliary parameter $s$, where $\rho_{l s}=\sqrt{\frac{10^{s}}{k_{0}}}$.

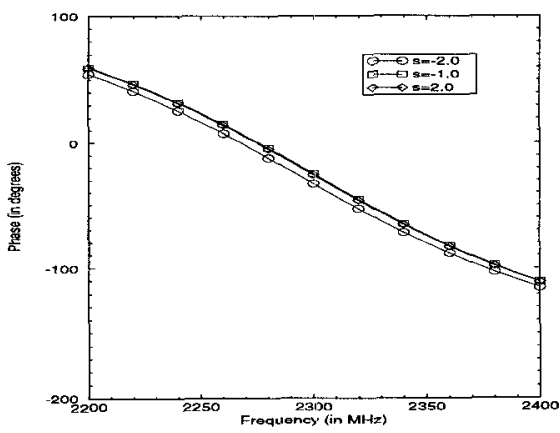

Figure 5: Phase of the input impedance of the patch antenna. 